\title{
Remoción de terceros molares mandibulares con asistencia endoscópica. Nota técnica de un nuevo procedimiento quirúrgico para prevenir lesiones del NAl y formación de defectos óseos
}

\author{
Removal of mandibular third molars with endoscopic approach. \\ Technical note of a new surgical procedure to avoid IAN damage \\ and bone defect formation
}

\author{
Fuentes $\mathrm{R}^{1}$, Beltrán $\mathrm{V}^{2}$, Cantín $\mathrm{M}^{3}$, Engelke $\mathrm{W}^{4}$
}

\begin{abstract}
RESUMEN
La variada posición anatómica de los terceros molares mandibulares presenta importantes desafíos asociados a su profundidad y grado de inclinación. Las complicaciones más habituales del procedimiento quirúrgico convencional de extracción se relacionan con la extensa osteotomía y poca visualización del sitio quirúrgico, que pueden generar consecuencias post-quirúrgicas como inflamación, dolor, trismus, lesiones reversibles e irreversibles del nervio alveolar inferior (NAl) o nervio lingual, riesgo de fractura y formación de defectos periodontales del segundo molar. La implementación de soportes rígidos en la óptica endoscópica ha permitido utilizar esta tecnología para realizar abordajes mínimamente invasivos para remover terceros molares mediante accesos flapless con una mínima osteotomía de la zona oclusal, conservando la pared bucal y lingual a través de la visualización directa y magnificada del sitio quirúrgico, adaptable a los movimientos del paciente durante la intervención. En este reporte se presenta un nuevo procedimiento quirúrgico mínimamente invasivo a través de asistencia endoscópica para la conservación ósea en la remoción de terceros molares mandibulares con riesgo de lesión del nervio alveolar inferior.
\end{abstract}

Rev. Clin. Periodoncia Implantol. Rehabil. Oral Vol. 5(2); 83-86, 2012.

Palabras clave: Endoscopio, terceros molares mandibulares, dientes impactados, osteotomía, daño al NAl.

\section{ABSTRACT}

Anatomic variability of the position of mandibular third molars represents significant challenges associated with its depth and angulation. The most common complications of conventional surgical procedure are related to extensive osteotomy and poor visualization, which can cause postsurgical effects such as inflammation, pain, trismus, reversible and irreversible lesions of the inferior alveolar nerve (IAN) or lingual nerve, fracture risk and formation of a deep periodontal defect on the distal aspect of the second molar. The implementation of rigid endoscopy in optics has allowed to use this technology via a minimally invasive approach to remove third molars by a minimally occlusal flapless ostectomy, preserving the buccal and lingual walls through direct and magnified visualization of the surgical site, adaptable to the patient's movements during the surgery. In this report, we present a new and minimally invasive procedure through endoscopic assistance for bone conservation in the removal of third molars at risk of inferior alveolar nerve injury

Rev. Clin. Periodoncia Implantol. Rehabil. Oral Vol. 5(2); 83-86, 2012.

Key words: Endoscope, mandibular third molars, impacted teeth, osteotomy, AIN injury.

\section{INTRODUCCIÓN}

Los terceros molares (3M) se presentan en el $90 \%$ de la población, y a lo menos un $33 \%$ de estos corresponden a $3 \mathrm{M}$ mandibulares impactados ${ }^{(1)}$. Su extracción es un procedimiento relativamente común, el que involucra manipulación quirúrgica de tejidos blandos y duros, donde el paciente usualmente presenta complicaciones y problemas post-operatorios.

La posición anatómica de los $3 \mathrm{M}$ mandibulares puede ser variable respecto a estructuras óseas como la rama mandibular y cuerpo mandibular, observándose incluso de forma ectópica(2). Algunas clasificaciones relacionan la posición de los $3 \mathrm{M}$ mandibulares con el eje del segundo molar adyacente o la profundidad y proximidad con la rama ascendente de la mandíbula, determinando diversos grados de inclusión ósea e impactación, mientras que otras toman en cuenta la posición de las raíces de los $3 \mathrm{M}$ y sus ápices con respecto al nervio alveolar inferior (NAI), que pueden ubicarse próximas, adyacentes o sobreproyactadas al canal mandibular y su nervio ${ }^{(3)}$. Para prevenir un eventual daño al NAl es necesario un estudio imagenológico adecuado a través de radiografías panorámicas o tomografía computarizada. La radiografía panorámica es el exámen más utilizado; otorga una adecuada información referente a la sobreproyección o proximidad al NAI de las raíces dentarias ${ }^{(4)}$.

Diversas técnicas han sido utilizadas para la remoción quirúrgica de $3 \mathrm{M}$ mandibulares incluidos o/e impactados, las cuales implican mayormente la eliminación del tejido óseo adyacente. Las complicaciones post-quirúrgicas generadas por osteotomía excesiva o rotura de periostio siguen siendo un gran problema y son generalmente representadas por inflamación, dolor, trismus, fracturas, daño accidental al NAI o al nervio lingual ${ }^{(5,6)}$.

1. Doctor Med. Dent. Departamento Integral del Adulto. Facultad de Odontología, Universidad de La Frontera. Temuco, Chile.

2. MSc. Especialista en Periodoncia e Implantología Oral. Departamento Integral del Adulto. Facultad de Odontología, Universidad de La Frontera. Temuco, Chile.

3. MSc. Programa de Doctorado en Ciencias Morfológica. Facultad de Medicina. Departamento Integral del Adulto. Facultad de Odontología, Universidad de La Frontera. Temuco, Chile.

4. PhD. Department of Maxillofacial Surgery. Goettingen University, Goettingen, Alemania.

Correspondencia autor: Ramón Fuentes. rfuentes@gmail.com. Departamento Integral del Adulto. Facultad de Odontología, Universidad de La Frontera. Calle Claro Solar $N^{\circ}$ 115-Of. 420. Temuco, Chile. Financiamiento: Esta investigación fue financiada con recursos institucionales del Departamento de Odontología Integral de la Universidad de La Frontera. Trabajo recibido el 25/05/2012. Aprobado para su publicación el 09/07/2012. 
En un cambio de paradigma hacia técnicas quirúrgicas atraumáticas para la remoción de $3 \mathrm{M}$ mandibulares, se han propuesto procedimientos como odontosección completa ${ }^{(7-9)}$, remoción parcial de coronas $^{(10)} \mathrm{o}$ extracción asistida por ortodoncia ${ }^{(11)}$. Si bien estas técnicas minimizan la cantidad de hueso a ser eliminado, algunas requieren la realización de dos intervenciones quirúrgicas ${ }^{(10,11)}$ y aún es necesario realizar amplios colgajos.

Actualmente, el modelo de cirugía sin colgajo (flapless), ha resultado exitoso en la remoción de dientes con dislocaciones horizontales ${ }^{(12)}$, por tanto, cambiar la clásica cirugía de $3 \mathrm{M}$ mediante colgajo lateral y osteotomía vestibular, por una cirugía sin colgajo con mantención completa de la tabla ósea bucal se ha transformado en una discusión substancial referente a procedimientos mínimamente invasivos.

La endoscopía es una herramienta de apoyo muy útil en la cirugía oral y máxilofacial mínimamente invasiva, con buenos resultados en diversos procedimientos como remoción de quistes, tratamiento de fracturas condilares y mandibulares, remoción de implantes desplazados al seno maxilar, elevación del seno maxilar, entre otros ${ }^{(13-15)}$. Aunque los endoscopios se asocian a instrumentos frágiles, el desarrollo soportes metálicos rígidos ha permitido aplicar la endoscopía en procedimientos de cirugía oral de pequeñas cavidades óseas como ocurre en la remoción de molares impactados. De esta forma, se mejora la visión del lecho quirúrgico y de zonas anatómicas de alta complejidad. Estas carácterísticas permiten disminuir considerablemente las complicaciones post-quirúrgicas versus procedimientos convencionales de remoción de $3 \mathrm{M}$ incluiodos e impactados, pues permite un acceso y osteotomía mínimanente invasiva ${ }^{(16)}$.

En este artículo se presenta una nueva técnica quirúrgica mínimamente invasiva a través de cirugía endoscópica flapless con conservación ósea para la remoción de $3 \mathrm{M}$ mandibulares impactados con riesgo de lesión del NAl y generar defectos óseos.

\section{RELATO DE CASOS}

Desde el servicio de Policlínicos de la Clínica Odontológica Asistencial Docente de la Universidad de La Frontera, fueron derivados al Centro de Microcirugía Oral dos pacientes de sexo femenino, de 18 y 30 años de edad para exodoncia de las pieza 4.8 y 3.8 , respectivamente, ambas por indicación ortodóncica. Durante la anamnesis remota, ambas pacientes relatan periodos intermitentes de inflamación de la zona retromolar, con dolor ocasional y molestias al comer. No se relataron antecedentes de enfermedad sistémica. En la anamnesis próxima ambas pacientes no relataron dolor ni inflamación. Previo a la realización de los procedimientos de remoción de $3 \mathrm{M}$, las pacientes aceptaron y firmaron un consentimiento informado de tratamiento.

En ambos casos se realizó una exodoncia mínimamente invasiva mediante asistencia endoscópica, con un endoscopio Karl Storz, con fibra óptica de $70^{\circ}$ de angulación (Figura 1A), y soportes metálicos rígidos de $2.7 \mathrm{~mm}$ (Figura 1B) y $1.9 \mathrm{~mm}$ (Figura 1C) de diámetro, que sirven de protección a la fibra óptica y permiten irrigar directamente la zona quirúrgica. De esta manera, el cirujano puede apoyarse a través del soporte en el lecho quirúrgico y a través de pequeños movimientos lograr una visualización completa del campo quirúrgico directo a través de una fuente de luz de $300 \mathrm{~W}$ con una capacidad de $6000 \mathrm{~K}^{(17)}$.

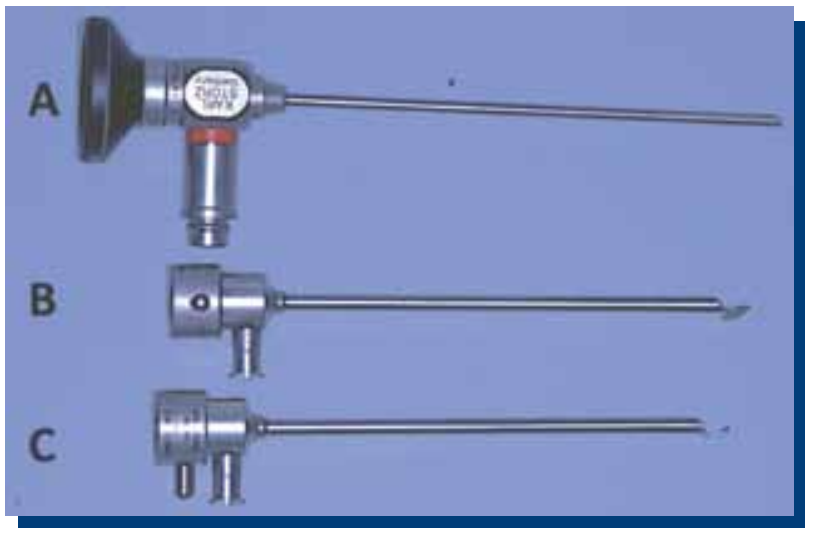

Figura 1. Protectores rígidos de diferentes diámetros, permiten dar soporte a la fibra óptica e irrigar la zona quirúrgica. Además protegen a la cámara en la zona quirúrgica durante el fresado y los procedimientos de odontosección. Diseñados por la empresa alemana Karl Storz. A: Fibra óptica. B: Soporte rígido de $2.7 \mathrm{~mm}$. C: Soporte rígido de $1.9 \mathrm{~mm}$.
En el caso 1, extra-oralmente la paciente no evidenció alteraciones de los tejidos blandos ni duros, sin relato de dolor ni de la alteración funcional en el momento. Intra-oralmente sólo se apreciaba una inflamación localizada de la región mucosa posterior de la zona retromolar. El paciente no presentaba enfermedad periodontal. El molar se encontraba en en mesioversión, con la región apical radicular proyectada sobre el canal mandibular, impactando la porción superior de la raíz distal del primer molar permanente (Figura 2A). La técnica quirúrgica consistió en un abordaje a través de una incisión mucosa lineal sin descarga para acceder a la superficie oclusal del $3 \mathrm{M}$, visualizando e sector endoscópicamente con el soporte de $2.7 \mathrm{~mm}$ (Figura 2B); luego con una fresa de carbide redonda $\mathrm{n}^{\circ} 4$ se seccionó verticalmente la corona del molar hasta llegar a la zona de furcación (Figura 2C). En ese momento apoyado por soporte metálico rígido, realizamos una sección vestíbulo-lingual de la porción coronaria del molar, hasta el límite óseo, Una vez finalizada la sección, con instrumental microquirúrgico de apoyo endoscópico, se fracturaron las secciones de corona en una mitad mesial y luego distal siendo fácilmente extraidas. Mediante elevadores finos y apoyo endoscópico se retiró la raíz distal y mesial a través de la cavidad oclusal sin realizar osteotomía vestibular (Figura 2D). Finalmente utilizando visualización endoscópica se revisó el lecho alveolar sano libre de restos apicales o detritus del fresado (Figura 2E). Se suturó con Vicril 4.0 atraumático y se dieron indicaciones postquirúrgicas al paciente.

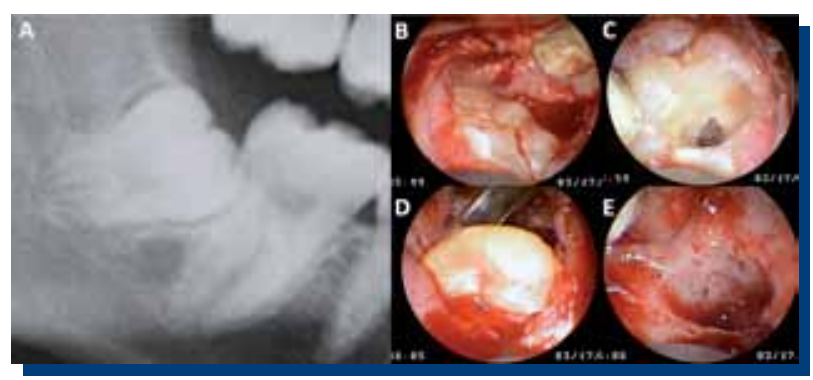

Figura 2. A. Radiografía diagnóstico del diente 4.8 con inclusión ósea y mucosa B. Osteotomía de la porción superior a la cara oclusal. C. Sección circular hasta la zona de furcación. D. Desplazamiento con microelevador de porción coronaria distal, y E. Alveolo limpio, se observan forámenes vasculares e indemnidad de la pared bucal ósea.

En el caso 2, el paciente no presentaba enfermedad periodontal, y se intervino un molar incluido en posición vertical con una leve inclinación vestibular respecto al eje longitudinal del segundo molar inferior izquierdo, con inclusión ósea y mucosa completa (Figura 3A). En este caso se realizó un una incisión lineal sin descarga para facilitar una osteotomía sólo en la porción oclusal del molar incluido y se procedió a un fresado con fresa redonda diamantada de $5 \mathrm{~mm}$ de diámetro, de bucal a lingual con visión directa endoscópica, sin comprometer en ningún momento el nervio lingual en relación estrecha a la porción lingual de la corona (Figuras 3B y $3 \mathrm{C}$ ). Se realizó la sección de la corona en una mitad mesial y distal con ayuda de un elevador fino (Figura 3D), y las secciones fueron fácilmete extraidas al igual que las raíces del diente. Finalmente se revisó endoscópicamente el lecho alveolar no observando remanentes del fresado óseo o restos de ápices no removidos (Figura 3E). Se suturó con Vicril 4.0 atraumático y se dieron indicaciones postquirúrgicas al paciente.

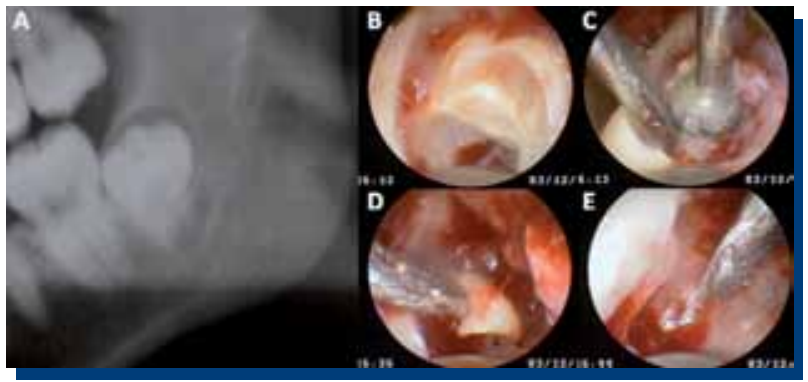

Figura 3. A. Radiografía diagnóstico del diente 3.8 con inclusión ósea y mucosa, relación estrecha al canal mandibular. B. Osteotomía y fresado sin comprometer la zona lingual de la corona en contacto con el nervio lingual. C. Fresado de la porción lingual con fresa diamantada redonda $n^{\circ} 4$. D. Desplazamiento de porción coronaria distal, y E. Alveolo limpio, remanente óseo indemne de todas las paredes. 
A los 7 días se realizó el retiro de sutura, donde los pacientes relataron dolor leve durante las primeras 48 horas con franca dismimución hacias las 72 horas. En ningún caso se relató edema extenso, trismus o pérdida de la sensibilidad en dientes, labio y/o lengua del lado donde se encontraba el molar impactado u otros signos compatibles con un daño neurosensorial postoperatorio.

\section{DISCUSIÓN}

Una de las principales complicaciones relacionadas con la extracción del $3 \mathrm{M}$ mandibular impactado es su estrecha relación con el NAI, que puede resultar en su daño neurosensorial temporal o permanente. La incidencia general de lesiones al NAI oscila entre el 1.3 a $5.3 \%$, elevándose al $19 \%$ si existe proximidad entre el NAI y las raíces del tercer molar ${ }^{(18)}$. Según Ortiz y San Pedro ${ }^{(3)}$ los $3 \mathrm{M}$ mandibulares en sujetos entre 15 y 25 años existe una prevalencia importante de raíces proyectadas sobre el NAI $(55.6 \%)$ seguido de molares con raíces adyacentes al NAI (25.6\%).

Convencionalmente, el procedimiento quirúrgico para la remoción de $3 \mathrm{M}$ mandibulares incluidos requiere la realización de un amplio colgajo seguido de una osteotomía oclusal y vestibular que permita la exposición de la corona, pero a menudo se hace necesaria una exposición más extensa hacia la raíz. Esto genera un gran trauma del tejido óseo y no reduce el posible riesgo de daño al NAI. Además, se genera una importante reducción de altura ósea, que origina un defecto óseo distal al segundo molar ${ }^{(19)}$, lo que influye en el estado periodontal, principalmente por una reducción en la profundidad de sondaje de la superficie distal del segundo molar ${ }^{(20-22)}$. Se han discutido diversas terapias cuando tenemos defectos periodontales asociados al segundo molar durante la cirugía del $3 \mathrm{M}^{(23)}$, a través del uso de injertos óseos o membranas reabsorbibles o no reabsorbibles para la cobertura del defecto $^{(24,25)}$. En este sentido, una técnica mínimamente invasiva con mayor conservación de tejido óseo se hace necesaria. El diseño del colgajo también ha sido discutido, donde pareciera que una técnica flapless tendría una influencia favorable en términos de la profundidad de sondaje al largo plazo ${ }^{(21,23)}$.

Para conservar el tejido óseo y reducir los riesgos de daño neurosensorial, se recomienda la sección y separación coronaria y radicular, lo que ha mostrado una menor morbilidad. Sin embargo, el difícil acceso y la escaza visibilidad lo hacen un procedimiento muy complejo y poco controlado.

La técnica presentada además de evitar la realización de colgajos amplios, también minimiza o evita la eliminación de tejido óseo, resultando en un procedimiento conservador. El uso de un acceso oclusal mediante endoscopía permite al cirujano visualizar en todo momento las estructuras internas y externas del diente lo que permite evitar o manejar situaciones inesperadas en la remoción del diente. Este enfoque conservador con asistencia endoscópica es fundamental cuando existe cercanía de estructuras anatómicas como el NAl, disminuyendo la posibilidad de lesionarlo; además evita el daño tisular extenso, minimiza la pérdida de sangre y reduce la morbilidad postoperatoria. Por otra parte, la exposición del nervio lingual es una situación menos frecuente, casi imposible de detectar en una radiografía panorámica o periapical previa, la cual puede observarse in situ desde una perspectiva intraalveolar con el endoscopio colocado dentro del alveolo. En el ámbito nacional, no existen estadísticas del daño al nervio lingual producto de la exodoncia de $3 \mathrm{M}$ incluidos, y los datos entregados corresponden a series extranjeras ${ }^{(5,6)}$. Sin ayuda visual seguiría siendo casi indetectable por el punto de vista clínico del cirujano ${ }^{(26)}$. Beltrán y cols. ${ }^{(26)}$ logran una magnificación del campo quirúrgico a través de la utilización de una óptica endoscópica de pequeño diámetro bajo una irrigación continua de suero a través del soporte rígido o inmersión endoscópica.

En nuestra experiencia, el uso del endoscopio como herramienta para realizar esta técnica microquirúrgica es de gran utilidad, ya que ofrece simultáneamente una visualización directa y magnificada del campo quirúrgico, junto a una buena iluminación ${ }^{(17)}$. Ventajas similares a esta técnica microquirúrgica han sido descritas por von Arx y cols. ${ }^{(27)}$ y Taschieri y cols. ${ }^{(28)}$ en otros procedimientos quirúrgicos. Una visualización intraoperatoria óptima es necesaria para lograr una alta tasa de éxito(28).

La técnica microquirúrgica descrita puede ser comparada inicialmente a un tratamiento endodóntico en analogía al acceso mediante trepanación y creación de un espacio interno en el diente como una cámara interna. Esto permite la movilización de partes de la corona y raíces, por lo que esta técnica puede ser denominada de "implosión" del diente. Además, según Engelke y cols. ${ }^{(19)}$ la eliminación del $3 \mathrm{M}$ inferior mediante un acceso oclusal microquirúrgico permite que el margen óseo bucal permanezca conservado sobre la unión cemento-esmalte del diente mesial y por lo tanto una separación menor del periostio en la exposición de la superficie del hueso, necesario como enfoque conservador.

En la literatura actual, nuevos procedimientos de extracción de $3 \mathrm{M}$ impactados o incluidos con un enfoque conservador han sido desarrollados por diversos autores. Landi y cols. ${ }^{(10)}$ presentaron una técnica para la extracción de $3 \mathrm{M}$ horizontales o en mesio-versión con estrecha relación al NAI mediante la remoción quirúrgica de la porción mesial de la corona anatómica del $3 \mathrm{M}$ para crear un espacio adecuado de migración mesial, y luego al menos de 6 meses, la extracción podría realizarse en una segunda sesión sin riego. En nuestra opinión es un enfoque interesante, sin embargo desventajas como el tiempo necesario, realización de 2 procedimientos quirúrgicos diferentes, riesgos de infección postoperatorias o riesgos de necrosis pulpar y posterior proceso infeccioso perirradicular en relación al NAI, poca utilidad ante una anatomía radicular compleja o riesgo de anquilosis son importantes de tener en cuenta, ya que la mayoría de los $3 \mathrm{M}$ impactados no cambian de posición en el tiempo. Además, no existen grandes ventajas técnicas de este procedimiento en comparación al descrito, ya que en ambos casos será necesario realizar una osteotomía oclusal mínima y sección coronal.

Tolstunov y cols. ${ }^{(29)}$ describieron una técnica de osteotomía pericoronaria, cuyo objetivo en una primera intervención es crear quirúrgicamente un espacio de erupción mediante la eliminación de todo el hueso ubicado sobre y alrededor de la corona, y luego luxar levemente el diente para aumentar su fuerza eruptiva inherente. Después de 6 a 8 semanas, en una segunda intervención, el diente en posición más coronal puede elevarse fácilmente con poco riesgo de lesión del nervio. Si bien esta técnica muestra resultado prometedores, presenta desventajas similares a la técnica de odontosección y migración reportada anteriormente ${ }^{(10)}$, además no es realizable en casos de impactaciones profundas.

Otra procedimiento conservador corresponde al uso de aparatos de ortodoncia para movilizar $3 \mathrm{M}$ lejos del NAl(11). Aunque con buenos resultados, también presenta variadas desventajas como el tiempo requerido, la aplicación de un dispositivo de ortodoncia en un área difícil de la boca junto a la compresión y la ulceración de los tejidos vecinos.

La técnica microquirúrgica endoscópica puede ser aplicada en casos de pericoronaritis, caries, o un defecto periodontal profundo, situaciones contraindicadas para las técnicas anteriormente descritas ${ }^{(10,11,29)}$, que deben ser realizadas sobre molares y tejidos sanos para reducir complicaciones infecciosas adicionales producto del tiempo involucrado (semanas o meses) y la cantidad de cirugías requeridas para cada $3 \mathrm{M}$. Por otro lado, la eliminación bajo visión directa endoscópica desde una aproximación oclusal puede tener una importancia considerable la reducción del riego de fractura mandibular por la pérdida de hueso como resultado de la osteotomía necesaria para la eliminar fragmentos de la raíz ubicados profundamente o en casos de raíces anquilosadas, ya que no existe necesidad de eliminación del hueso lateral ${ }^{(19)}$, siendo probablemente la única técnica que en la actualidad ofrezca esta ventaja.

Además, esta técnica es de tipo flapless. Kim y cols. ${ }^{(12)}$ demostraron que el dolor postoperatorio, inflamación y profundidad del defecto de bolsa distal al segundo molar son significativamente menores al realizar una extracción de $3 \mathrm{M}$ sin colgajos amplios. Aunque se han diseñado diversos tipos de colgajos para la extracción de $3 \mathrm{M}$, en mayor o menor medida muestran presencia de dolor continuo, dolor en la apertura máxima, edema, y todos generan trismus. El uso de incisiones sin descargas o pedículos mejora aspectos de la experiencia del dolor postoperatorio y reduce la incidencia de osteítis alveolar ${ }^{(30)}$.

Respecto a la duración de la cirugía, esta depende de la complejidad y grado de formación del cirujano, con un promedio de 10-20 minutos. Un tiempo quirúrgico adicional es requerido en casos complejos como en riesgo de fractura de la raíz o en casos de anquilosis.

Si bien existen gran cantidad de ventajas en relación a los procedimientos actuales, el procedimiento microquirúrgica descrito no está exento de algunos requerimientos y desafíos asociados. En primer lugar la técnica requiere un equipo básico de endoscópia y la instrucción 
especial del cirujano. El endoscopio ofrece una imagen ampliada en un monitor de vídeo a distancia de dos dimensiones, por lo que requiere el desarrollo de una coordinación óculo-manual específica, con amplia comprensión del concepto tridimensional de la anatomía quirúrgica oral y maxilofacial ${ }^{(16)}$. En segundo lugar se ha limitado su uso cuando el objeto de remoción es de gran tamaño(15), situación que es superada con la sección del objeto, gracias a las misma ventajas de visualización que ofrece la endoscopia. Tercero, al utilizar un equipo especial, se requiere de una capacitación técnica del personal quirúrgico de apoyo y la preparación del endoscopio en el momento de la cirugía. Finalmente, debido a la alta precisión requerida y procedimiento paso a paso, el tiempo necesario para realizar el procedimiento, el tiempo utilizado puede ser mayor en comparación con el procedimiento quirúrgico convencional, dependiente del grado de impactación, angulación del diente, morfología radicular, presencia de anquilosis y relación de las raíces del molar con el canal mandibular. Además, la curva de aprendizaje del cirujano y los costos iniciales de los equipos puede limitar su aplicación en comparación al procedimiento tradicional.

$\mathrm{Si}$ definimos como medidas de éxito en la remoción de dientes complejos la conservación de las estructuras óseas y los tejidos blandos que lo cubren, esta técnica puede ser importante avance en el área quirúrgica, pero se renuncia deliberadamente a la eliminación de dientes anatómicamente intactos. El uso de la endoscopia en el área cráneo-maxilofacial ha permitido mejorar la capacidad intraoperatoria con una visualización clara y magnificada del campo operatorio, junto a una buena iluminación de las estructuras, permitiendo realizar una cirugía mínimamente invasiva, conservadora, con una disección precisa y sin colgajo. Estas características hacen que esta técnica puede ser considerada como un enfoque alternativo para la extracción de $3 \mathrm{M}$ en las situaciones anatómicas más complejas de la anatomía radicular, remoción de fragmentos radiculares o raíces anquilosadas que pueden ser realizados sin osteotomía desde un acceso oclusal o en casos de proximidad al NAI. Todo esto se traduce en complicaciones postquirúrgicos mínimas, como menor inflamación, hemorragia o lesiones nerviosas y también menor riego de fracturas. Es conveniente plantear un estudio del tipo Ensayo Clínico, para conocer la real efectividad de esta nueva intervención en relación a la cirugía convencional.

Finalmente, esta técnica nos entrega una ventaja comparativa a todos las técnicas quirúrgicas existentes como es la visualización completa y detallada de los lechos óseos post-extracción lo que permite mantener indemne la pared ósea bucal, realizar curetajes con visualización directa, evitando remantes de ápices alveolares, quistes residuales, tejido pericoronario o remanentes del fresado óseo o dentario. El apoyo endoscópico en cirugía oral es una herramienta para el tratamiento predecible y con mínimas complicaciones post-quirúrgicas para el paciente.

\section{CONFLICTOS DE INTERÉS}

Los autores declaran no tener ninguna vinculación financiera en relación con cualquiera de los productos involucrados en este estudio.

\section{REFERENCIAS BIBLIOGRÁFICAS}

1. Scherstén E, Lysell L, Rohlin M. Prevalence of impacted third molars in dental students. Swed Dent J, 1989; 13: 7-13.

2. Fuentes FR, Oporto VG. Tercer molar ectópico impactado en zona retromolar. Reporte de caso. Int J Morphol, 2009; 27: 35-38.

3. Ortiz CP, San Pedro VJ. Relationship between the mandibular canal and the roots of the mandibular third molars of young patients in Talca. Int $\mathrm{J}$ Odontostomat, 2009; 3: 131-135.

4. Tantanapornkul W, Okouchi K, Fujiwara $Y$ y cols. A comparative study of conebeam computed tomography and conventional panoramic radiography in assessing the topographic relationship between the mandibular canal and impacted third molars. Oral Surg Oral Med Oral Pathol Oral Radiol Endod, 2007; 103: 253-259.

5. Shepherd JP, Brickley M. Surgical removal of third molars. BMJ, 1994; 309: 620621.

6. Meisami T, Sojat A, Sàndor GK, Lawrence HP, Clokie CM. Impacted third molars and risk of angle fracture. Int J Oral Maxillofac Surg, 2002; 31: 140-144.

7. Genú PR, Vasconcelos BC. Influence of the tooth section technique in alveolar nerve damage after surgery of impacted lower third molars. Int $J$ Oral Maxillofac Surg, 2008; 37: 923-928.

8. Arakeri G, Arali V. Tooth section technique and pain upon elevation in third molar removal. Int J Oral Maxillofac Surg, 2010; 39: 98-99.

9. Ngeow WC. Tooth section technique for wisdom teeth. Int J Oral Maxillofac Surg, 2009; 38: 908

10. Landi L, Manicone PF, Piccinelli S, Raia A, Raia R. A novel surgical approach to impacted mandibular third molars to reduce the risk of paresthesia: A case series. $J$ Oral Maxillofac Surg, 2010; 68: 969-974.

11. Bonetti GA, Parenti SI, Checchi L. Orthodontic extraction of mandibular third molar to avoid nerve injury and promote periodontal healing. J Clin Periodontol, 2008; 35: 719-723.

12. Kim HR, Choi BH, Engelke W, Serrano D, Xuan F, Mo DY. A comparative study on the extractions of partially impacted mandibular third molars with or without a buccal flap: A prospective study. J Oral Maxillofac Surg, 2011; 69: 966-970.

13. Kahairi A, Ahmed Khan S, Amirozi A. Endoscopic-assisted enucleation of radicular cysts - A case report. Malays J Med Sci, 2010; 17: 56-59.

14. Schön R, Gutwald R, Schramm A, Gellrich NC, Schmelzeisen R. Endoscopyassisted open treatment of condylar fractures of the mandible: Extraoral vs. intraoral approach. Int J Oral Maxillofac Surg, 2002; 31: 237-243.

15. Nakamura N, Mitsuyasu T, Ohishi M. Endoscopic removal of a dental implant displaced into the maxillary sinus: Technical note. Int J Oral Maxillofac Surg, 2004; 33: 195-197.

16. Suarez-Cunqueiro MM, Schoen R, Schramm A, Gellrich NC, Schmelzeisen R Endoscopic approach to removal of an ectopic mandibular third molar. $\mathrm{Br} \mathrm{J}$ Oral Maxillofac Surg, 2003; 41: 340-342.
17. Engelke W, Galle C. Endoscopic Interventions in oral implantology - A report of 5 years clinical experience. Int J Odontostomat, 2008; 2: 153-161.

18. Renton T, Hankins M, Sproate C, McGurk M. A randomised controlled clinical trial to compare the incidence of injury to the inferior alveolar nerve as a result of coronectomy and removal of mandibular third molars. Br J Oral Maxillofac Surg, 2005; 43: 7-12.

19. Engelke W, Bierbaum J, Choi EJ. Microsurgical removal of inferior third molars using an occlusal approach. Dtsch Zahnärztl Z, 2011; 66: 415-423.

20. Quee TA, Gosselin D, Millar EP, Stamm JW. Surgical removal of the fully impacted mandibular third molar. The influence of flap design and alveolar bone height on the periodontal status of the second molar. J Periodontol, 1985; 56: 625-630.

21. Szmyd L, Hester WR. Crevicular depth of the second molar in impacted third molar surgery. J Oral Surg. Anaesth Hosp Dent Surv, 1963; 21: 185-189.

22. LaBelle RE. The influence of third molars on periodontal health. Minneap Dist Dent J, 1969; 53: 25-28.

23. Peng KY, Tseng YC, Shen EC, Chiu SC, Fu E, Huang YW. Mandibular second molar periodontal status after third molar extraction. J Periodontol, 2001; 72: 16471651.

24. Sammartino G, Tia M, Bucci T, Wang HL. Prevention of mandibular third molar extraction-associated periodontal defects: A comparative study. J Periodontol, 2009; 80: 389-396

25. Corinaldesi G, Lizio G, Badiali G, Morselli-Labate AM, Marchetti C. Treatment of intrabony defects after impacted mandibular third molar removal with bioabsorbable and non-resorbable membranes. J Periodontol, 2011; 82: 1404-1413.

26. Beltrán V, Fuentes R, Engelke W. Endoscopic visualization of anatomic structures as a support tool in oral surgery and implantology. J Oral Maxillofac Surg, 2012; 70: e1-6.

27. von Arx T, Montagne D, Zwinggi C, Lussi A. Diagnostic accuracy of endoscopy in periradicular surgery - A comparison with scanning electron microscopy. Int Endod J, 2003; 36: 691-699.

28. Taschieri S, Del Fabbro M, Testori T, Weinstein R. Microscope versus endoscope in root-end management: a randomized controlled study. Int J Oral Maxillofac Surg 2008; 37: 1022-1026.

29. Tolstunov L, Javid B, Keyes L, Nattestad A. Pericoronal ostectomy: An alternative surgical technique for management of mandibular third molars in close proximity to the inferior alveolar nerve. J Oral Maxillofac Surg, 2011; 69: 1858-1866.

30. Goldsmith SM, De Silva RK, Tong DC, Love RM. Influence of a pedicle flap design on acute postoperative sequelae after lower third molar removal. Int $J$ Oral Maxillofac Surg, 2012; 41: 371-375. 